CONCISE REPORT

\title{
Raised plasma levels of asymmetric dimethylarginine are associated with cardiovascular events, disease activity, and organ damage in patients with systemic lupus erythematosus
}

\author{
I E M Bultink, T Teerlink, J A Heijst, B A C Dijkmans, A E Voskuyl
}

Ann Rheum Dis 2005;64:1362-1365. doi: 10.1136/ard.2005.036137

Background: Asymmetric dimethylarginine (ADMA) is an endogenous nitric oxide inhibitor and a new independent risk factor for endothelial dysfunction and cardiovascular disease.

Objective: To investigate the relationship between plasma ADMA levels and cardiovascular events (CVEs) and disease characteristics in patients with systemic lupus erythematosus (SLE).

Methods: Demographic and clinical data were collected and plasma ADMA levels were measured in 107 patients with SLE. A modified organ damage index was calculated as defined by the Systemic Lupus International Collaborating Clinics/American College of Rheumatology Damage Index (SDI), excluding CVE as an item.

Results: Cardiovascular disease, defined as $\geqslant 1$ previous arterial CVE, was recorded in 16/107 (15\%) patients with SLE and increased across tertiles of ADMA levels ( $p=0.023$ for trend). Mean plasma ADMA levels were significantly higher in patients with SLE with a history of CVEs than in patients without a CVE history $(p=0.018)$. In multiple regression analysis a high SLEDAl score, high modified $\mathrm{SDI}$, high titre of anti-dsDNA antibodies, and low serum HDL were significantly associated with high plasma ADMA levels. Conclusion: In patients with SLE, plasma ADMA levels are significantly associated with CVEs, measures of disease activity, and organ damage, independently of an unfavourable lipid profile.

\footnotetext{
C
} ardiovascular disease, including coronary heart disease, ${ }^{1}$ ischaemic cerebrovascular disease, ${ }^{2}$ and peripheral vascular disease ${ }^{3}$ has been recognised as an important cause of morbidity and mortality in patients with systemic lupus erythematosus (SLE). The mechanisms underlying the accelerated atherosclerosis in SLE are not completely clear because the traditional risk factors fail to account fully for the excess of cardiovascular events (CVEs) in lupus patients. ${ }^{4}$

Asymmetric dimethylarginine (ADMA) is an endogenous inhibitor of nitric oxide synthase $\mathrm{e}^{5}$ and is associated with endothelial dysfunction. ${ }^{6}$ Furthermore, high ADMA plasma levels are a risk factor for acute coronary events ${ }^{7}$ and a predictor of mortality and CVEs in patients with end stage renal disease. ${ }^{8}$

In the presence of anti-dsDNA, up regulation of methylation of arginine residues in proteins has been demonstrated in vitro. ${ }^{9}$ As ADMA is released upon proteolysis of methylated proteins, ${ }^{5}$ anti-dsDNA antibodies may be a trigger for enhanced ADMA production in SLE. However, this has not been studied in vivo.

This study aimed at assessing the hypothesis that plasma ADMA levels are associated with CVEs in patients with SLE, and with the presence of anti-dsDNA and other lupus characteristics

\section{METHODS}

\section{Data collection and clinical measures}

One hundred and seven consecutive patients fulfilling the revised criteria for the classification of SLE were included. The local ethics committee approved the study. All patients provided informed consent. Demographic and clinical characteristics were systematically documented by questionnaire, chart review, and clinical examination. Data collection comprised documented previous arterial CVEs. Coronary artery events were defined as myocardial infarction, coronary artery by-pass surgery, coronary angioplasty/stenting, and angina pectoris. Ischaemic cerebrovascular events were defined as transient ischaemic attacks, ischaemic stroke, or carotid endarterectomy. Peripheral artery events were defined as peripheral grafting or symptomatic peripheral artery ischaemia, confirmed by angiography. Disease activity was measured by the SLE Disease Activity Index (SLEDAI) and European Consensus Lupus Activity Measure (ECLAM). A modified organ damage index was calculated as defined by the Systemic Lupus International Collaborating Clinics/ American College of Rheumatology Damage Index (SDI), excluding CVEs as a damage item.

\section{Biochemical measurements}

ADMA was measured by high performance liquid chromatography, as published previously. ${ }^{10}$ The upper limit of the reference range is $0.55 \mu \mathrm{mol} / \mathrm{l}$. The subjects had fasted and had refrained from smoking and alcohol consumption for at least 24 hours before sampling. Laboratory investigations at the time of ADMA measurement included $\mathrm{C}$ reactive protein, serum creatinine, immunological measures, and fasting levels of blood glucose, plasma homocysteine, serum total cholesterol, high density lipoprotein cholesterol and triglycerides. Anti-dsDNA titres were evaluated using an indirect immune fluorescence technique with Crithidia luciliae as

Abbreviations: ADMA, asymmetric dimethylarginine; CVE, cardiovascular event; DDAH, dimethylarginine-

dimethylaminohydrolase; hnRNP, heterogeneous nuclear ribonucleoprotein; PRMT, protein arginine methyltransferase; SDI, Systemic Lupus International Collaborating Clinics/American College of Rheumatology Damage Index; SLE, systemic lupus erythematosus; SLEDAI, SLE Disease Activity Index 
Table 3 Multivariate analysis of variables associated with plasma ADMA levels

\begin{tabular}{|c|c|c|c|c|}
\hline Variables & B & $95 \% \mathrm{Cl}$ & Standardised B & p Value \\
\hline SLEDAI & 0.0073 & 0.004 to 0.011 & 0.340 & $<0.001$ \\
\hline Titre of anti-dsDNA (IE/ml) & 0.0003 & 0.0001 to 0.001 & 0.250 & 0.006 \\
\hline SDI (modified) & 0.0097 & 0.002 to 0.018 & 0.190 & 0.019 \\
\hline Serum HDL (mmol/l) & -0.0004 & -0.078 to -0.003 & -0.171 & 0.037 \\
\hline
\end{tabular}

(0.44 (0.09) $\mu \mathrm{mol} / \mathrm{l}, \mathrm{p}=0.018)$. Figure $\mathrm{l}$ shows that the percentage of patients with SLE with previous CVEs increased across the tertiles of plasma ADMA levels $(p=0.023$ for trend $)$. Traditional risk factors for arterial cardiovascular disease as well as ADMA levels were not significantly associated with previous CVEs in multiple regression analyses (data not shown).

\section{Variables associated with plasma ADMA levels}

Table 2 shows the results of univariate analyses.

In a stepwise multiple regression analysis a high SLEDAI score, high modified SDI, high titre of anti-dsDNA antibodies, and low serum HDL were significantly and independently associated with plasma ADMA levels (table 3). The Pearson correlation coefficient between the SLEDAI score and ADMA levels was $0.503(\mathrm{p}<0.01)$.

\section{DISCUSSION}

The main finding of this study is that high plasma ADMA levels were significantly associated with CVEs in patients with SLE. In addition, ADMA levels were significantly associated with measures of disease activity and organ damage. As far as we know, this is the first study of the association between ADMA levels and CVEs and disease characteristics in patients with SLE.

The increased mean ADMA level in the group of patients with SLE with a history of CVEs is in agreement with studies in other patient groups at high risk of the development of cardiovascular disease. Previous studies demonstrated increased oxidative stress in $\mathrm{SLE}^{11}$ as well as raised plasma levels of circulating oxidised low density lipoprotein in patients with SLE with a history of CVEs. ${ }^{12}$ The major route of ADMA elimination is degradation by the enzyme dimethylarginine-dimethylaminohydrolase (DDAH), which is very sensitive to oxidative stress. ${ }^{13}$ Reduced DDAH activity by increased oxidative stress may thus contribute to increased ADMA levels in SLE.

The second important finding of our study is the association between ADMA levels and measures of disease activity, especially a high titre of anti-dsDNA antibodies. This observed association is in line with results of in vitro studies. Anti-dsDNA antibodies were shown to be reactive with the arginine-glycine-rich domains in recombinant heterogeneous nuclear ribonucleoprotein A2 (hnRNP A2). ${ }^{9}$ Remarkably, these domains are also preferred sites for the methylation of arginine to ADMA by type 1 protein arginine methyltransferase (PRMTl).$^{14}$ In the presence of anti-dsDNA, methylation of hnRNP A2 by PRMT1 was increased to 3.5 times that of the control level. Therefore, anti-dsDNA antibodies may be a trigger for increased ADMA production by up regulating methylation of arginine residues by PRMT1. Moreover, antidsDNA monoclonal antibodies enhance the inflammatory reaction by the release of proinflammatory cytokines from mononuclear cells. ${ }^{15}$ These studies and our findings provide scientific rationale for the hypothesis that anti-dsDNA antibodies may have a role in the development of cardiovascular disease in SLE by enhancing ADMA production and by augmenting the inflammatory reaction.
Limitations of our study include the relatively small study group and the cross sectional design. In our study, raised ADMA levels and traditional risk factors for CVEs were not independently associated with previous CVEs in multiple regression analysis. This finding might be explained by the relatively small study group and number of previous CVEs. Furthermore, cross sectional data do not allow causality to be established. A prospective study in a larger study group is required to answer definitively the question of whether raised ADMA levels are an independent risk factor for CVEs in patients with SLE.

The association between ADMA levels and modified SDI (excluding CVEs as an item) suggests that the nitric oxide pathway might also be involved in the development of damage in other organ systems in SLE. Further studies are advocated to elucidate the role of the nitric oxide pathway and its endogenous inhibitor ADMA in lupus pathogenesis and the development of organ damage in SLE.

\section{ACKNOWLEDGEMENT}

We thank Sigrid de Jong for the skilful determination of ADMA.

\section{Authors' affiliations}

I E M Bultink, B A C Dijkmans, A E Voskuyl, Department of Rheumatology, VU University Medical Centre, Amsterdam, The Netherlands

T Teerlink, J A Heijst, Department of Clinical Chemistry, VU University Medical Centre, Amsterdam, The Netherlands

Correspondence to: Dr I E M Bultink, Department of Rheumatology, VU University Medical Centre, Postbus 7057, 1007 MB, Amsterdam, The Netherlands; iem_bultink@hotmail.com

Accepted 4 February 2005

\section{REFERENCES}

1 Manzi S, Meilahn EN, Rairie JE, Conte CG, Medsger TA Jr, Jansen$\mathrm{McWilliams} \mathrm{L}$, et al. Age-specific incidence rates of myocardial infarction and angina in women with systemic lupus erythematosus: comparison with the Framingham Study. Am J Epidemiol 1997;145:408-15.

2 Kitagawa Y, Gotoh F, Koto A, Okayasu H. Stroke in systemic lupus erythematosus. Stroke 1990;21:1533-9.

3 McDonald J, Stewart J, Urowitz MB, Gladman DD. Peripheral vascular disease in patients with systemic lupus erythematosus. Ann Rheum Dis 1992;51:56-60.

4 Esdaile JM, Abrahamowicz M, Grodzicky T, Li Y, Panaritis C, du Berger R, et al. Traditional Framingham risk factors fail to fully account for accelerated atherosclerosis in systemic lupus erythematosus. Arthritis Rheum 2001;44:2331-7.

5 Vallance P. Importance of asymmetrical dimethylarginine in cardiovascular risk. Lancet 2001;358:2096-7.

6 Boger RH, Bode-Boger SM, Szuba A, Tsao PS, Chan JR, Tangphao O, et al. Asymmetric dimethylarginine (ADMA): a novel risk factor for endothelial dysfunction: its role in hypercholesterolemia. Circulation 1998;98:1842-7.

7 Valkonen VP, Paiva H, Salonen JT, Lakka TA, Lehtimaki T, Laakso J, et al. Risk of acute coronary events and serum concentration of asymmetrical dimethylarginine. Lancet 2001;358:2127-8.

8 Zoccali C, Bode-Boger S, Mallamaci F, Benedetto F, Tripepi G, Malatino L, et al. Plasma concentration of asymmetrical dimethylarginine and mortality in patients with end-stage renal disease: a prospective study. Lancet 2001;358:2113-17.

9 Sun KH, Tang SJ, Wang YS, Lin WJ, You RI. Autoantibodies to dsDNA crossreact with the arginine-glycine-rich domain of heterogeneous nuclear ribonucleoprotein A2 (hnRNP A2) and promote methylation of hnRNP A2. Rheumatology (Oxford) 2003;42:154-61. 
10 Teerlink T, Nijveldt RJ, de Jong S, van Leeuwen PA. Determination of arginine, asymmetric dimethylarginine, and symmetric dimethylarginine in human plasma and other biological samples by high-performance liquid chromatography. Anal Biochem 2002;303:131-7.

11 Nuttall SL, Heaton S, Piper MK, Martin U, Gordon C. Cardiovascular risk in systemic lupus erythematosus - evidence of increased oxidative stress and dyslipidaemia. Rheumatology (Oxford) 2003;42:758-62.

12 Svenungsson $E$, Jensen-Urstad K, Heimburger M, Silveira A, Hamsten A, de Faire $U$, et al. Risk factors for cardiovascular disease in systemic lupus erythematosus. Circulation 2001;104:1887-93.
13 Tran $C T$, Leiper JM, Vallance $P$. The DDAH/ADMA/NOS pathway. Atheroscler Suppl 2003;4:33-40.

14 Gary JD, Clarke S. RNA and protein interactions modulated by protein arginine methylation. Prog Nucleic Acid Res Mol Biol 1998:61:65-131.

15 Sun KH, Yu CL, Tang SJ, Sun GH. Monoclonal anti-double-stranded DNA autoantibody stimulates the expression and release of IL-1 beta, IL-6, IL-8, IL-10 and TNF-alpha from normal human mononuclear cells involving in the lupus pathogenesis. Immunology 2000;99:352-60

\section{$\mathrm{ECHO}$}

\section{Rituximab induces remission in stiff person syndrome}

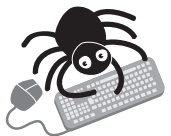

Please visit the Annals of the Rheumatic Diseases website [www. annrheumdis. com] for a link to the full text of this article.
$\mathrm{T}$

he first report of successful treatment with a monoclonal anti-B cell antibody may offer new hope for patients with stiff person syndrome, a rare but ultimately fatal autoimmune disease of the CNS.

Monoclonal antibody specific for B cells expressing CD20 antigen (rituximab) alleviated severe symptoms when other treatments failed. It abolished intrathecal autoantibody against glutamic acid decarboxylase (GAD), suggesting that the syndrome is a $\mathrm{B}$ cell mediated autoimmune disease.

The 41 year old woman was an emergency admission in January 2004 with prolonged painful muscle spasms in her neck and back and arms and legs, rendering her bedridden and dependent on carers for months previously. She was taking baclofen and dantrolene sodium daily, fentanyl patches twice weekly and parenteral diazepam up to $80 \mathrm{mg}$ and diamorphine up to $25 \mathrm{mg}$ daily, providing subjective benefit.

The syndrome had been diagnosed in 2001. Various antispasmodic agents and disease modifying treatments, including seven courses of intravenous immunoglobulin and courses of cytotoxic drugs, tried since then had had no lasting success. Eventually, intrathecal infusions of hydrocortisone produced transient improvement, in December 2003.

However, after just over two weeks of rituximab at $375 \mathrm{mg} / \mathrm{m}^{3}$, in January 2004, muscle spasms started to subside, and the woman was able to sit up and shower herself for the first time in two years. Testing in November 2003 showed intrathecal autoantibody to GAD, but at 17 days' treatment none was evident. One month after discharge her condition was stable and she needed only small doses of oral benzodiazepine and analgesia until symptoms recurred, at six weeks, when she was given further rituximab, with mycophenolate mofetil. She improved again after 14 days and was discharged, remaining well after five months.

Symptomatic treatment relies on $\gamma$-amino butyric acid (GABA) enhancing agents, but previous treatments modifying immune response by reducing antibody to GAD, a rate limiting enzyme in GABA synthesis, has had variable results.

A Baker MR, et al. Journal of Neurology, Neurosurgery, and Psychiatry 2005;76:999-1001. 